\title{
Determinação Visual do Componente Rápido do Excesso do Consumo de Oxigênio Após o Exercício
}

\section{Visual Determination of the Fast Component of Excessive Oxygen Uptake After Exercise}

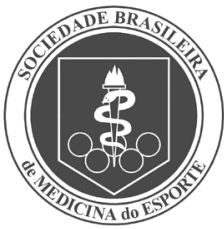

Artigo Original

Rômulo Cássio de Moraes Bertuzzi Adriano Eduardo Lima Silva1,2 Flávio de Oliveira Pires

Maria Augusta Peduti Dal'Molin Kiss'

1.Escola de Educação Física e Esporte da Universidade de São Paulo, São Paulo.

2.Grupo de Pesquisa em Ciências do Esporte. Universidade Federal de Alagoas, Maceió, Alagoas.

Endereço para correspondência: Rua Clorindo de Oliveira Cajé, 91. 05371-140 - São Paulo, SP E-mail: bertuzzi@usp.br

\section{RESUMO}

O objetivo do presente estudo foi analisar a validade, a reprodutibilidade e a objetividade do método de inspeção visual durante a identificação da fase rápida do excesso do consumo de oxigênio após o exercício (EPOC RÁPIDO). Dez homens fisicamente ativos (idade de 23,0 $\pm 4,0$ anos, estatura de 176,4 $\pm 6,8 \mathrm{~cm}$, massa corporal de $72,4 \pm 8,2 \mathrm{~kg}, \dot{V} \mathrm{O}_{2} \max 3,0 \pm 0,5 \mathrm{~L} \cdot \mathrm{min}^{-1}$ ) realizaram um teste incremental máximo e um teste de carga constante até a exaustão a $110 \%$ da carga máxima obtida no teste incremental. O consumo de oxigênio foi mensurado respiração a respiração durante dez minutos de recuperação passiva após o teste de carga constante. O EPOC RÁPIDO foi determinado matematicamente e visualmente por três avaliadores. $O$ método visual foi aplicado duas vezes nos três avaliadores para verificar a sua reprodutibilidade. Não foram detectadas diferenças significativas entre os valores do $E P O C_{\text {RAPIDO }}$ estabelecidos pelo método matemático $(0,98 \pm 0,45 \mathrm{~L})$ e pelo método visual identificado pelos três avaliadores $(1,04 \pm 0,45 \mathrm{~L}, 1,02 \pm 0,45 \mathrm{~L}$ e1,02 \pm $0,45 \mathrm{~L})$. Nenhuma diferença foi encontrada entre a primeira e a segunda identificação feita pelos avaliadores (avaliador 1: 1,04 \pm 0,45L vs 1,04 \pm 0,49L; avaliador 2: 1,02 $\pm 0,45 \mathrm{~L}$ vs 1,01 $\pm 0,44 \mathrm{~L}$ e avaliador 3: 1,02 $\pm 0,45 \mathrm{~L}$ vs $1,03 \pm 0,47 \mathrm{~L})$. Além disso, o coeficiente de correlação intraclasse entre as duas identificações foi alto para todos os avaliadores (ICC entre 0,97 e0,99). Esses resultados sugerem que a inspeção visual é um método válido, objetivo e reprodutivo para a estimativa do EPOC RápiDo.

Palavras-chave: débito alático de oxigênio, EPOC, teste anaeróbio, validade, exercício supramáximo.

\section{ABSTRACT}

The objective of this study was to analyze the validity, reproducibility and objectivity of the visual inspection method during the identification of the fast component of excess post-exercise oxygen consumption $\left(\mathrm{EPOC}_{\mathrm{FAST}}\right.$ ). Ten healthy physically active men (age $=23.0+4.0$ years; height $=176.4+6.8 \mathrm{~cm}$; body mass $=72.4+8.2 \mathrm{~kg} ; V_{2} \mathrm{O}_{2 \mathrm{MAX}}=3.0+0.5{\mathrm{~L} \cdot \mathrm{min}^{-1}}^{-1}$ ) performed a maximal incremental exercise and a constant workload test until exhaustion corresponding to $110 \%$ of maximal workload reached during the maximal incremental exercise. Oxygen consumption was measured breath-by-breath for 10 minutes during the passive recovery after the constant workload test. EPOC FAST was mathematically and visually determined by three evaluators. Double visual determination of EPOC FAST was carried out by each evaluator for reproducibility determination. There were no significant differences between EPOCFAST values obtained by mathematical $(0.98 \pm 0.45 \mathrm{~L})$ or visual method $(1.04 \pm 0.45 \mathrm{~L} ; 1.02 \pm 0.45 \mathrm{~L}$ and $1.02 \pm 0.45 \mathrm{~L})$. None significant difference was found between the first and second visual assessment carried out by the evaluators (evaluator 1: 1.04 $\pm 0.45 \mathrm{~L}$ vs $1.04 \pm 0.49 \mathrm{~L}$; evaluator 2: $1.02 \pm 0.45 \mathrm{~L}$ vs $1.01 \pm 0.44 \mathrm{~L}$ and evaluator 3: 1.02 $\pm 0.45 \mathrm{~L}$ vs $1.03 \pm 0.47 \mathrm{~L}$ ). Finally, coefficient of intra-class correlation between determinations was high for all evaluators (ICC from 0.97 to 0.99 ). These results suggest that the visual method is valid, objective and reproducible for determination of the EPOC FAST.

Keywords: alactic oxygen debt, EPOC, anaerobic test, validity, supramaximal exercise. 


\section{INTRODUÇÃO}

O estabelecimento do perfil bioenergético durante o exercício é uma das principais formas de caracterizar a demanda imposta pelos esportes e, consequentemente, aumentar a especificidade na prescrição do treinamento físico. Tem-se sugerido que durante o exercício físico realizado em intensidades abaixo do consumo máximo de oxigênio ( $\mathrm{V}_{2} \mathrm{max}$ ), o metabolismo aeróbio é o principal responsável pelo fornecimento de energia para contração muscular ${ }^{(1)}$. Portanto, a medida do consumo de oxigênio $\left(\dot{\mathrm{V}}_{2}\right)$ pode ser utilizada para representar a contribuição do metabolismo aeróbio durante o exercício realizado abaixo do $\dot{V}_{2} \mathrm{max}^{(2)}$. Por outro lado, em intensidades acima do $\dot{\mathrm{V}}_{2} \max$, a demanda energética excede a potência aeróbia máxima ${ }^{(3)}$, o que torna fundamentais as participações dos metabolismos anaeróbios lático e alático(4).

Diversos testes foram propostos para avaliar a contribuição de cada um dos metabolismos anaeróbios em exercício supra-Vं $\mathrm{O}_{2}$ max $^{(5)}$. Entre os principais testes, destaca-se a fase rápida do excesso do $\dot{\mathrm{V}}_{2}$ após

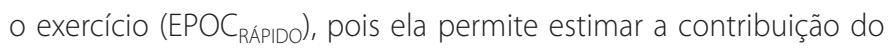
metabolismo anaeróbio alático (MAA) em situações laboratoriais ${ }^{(6)} \mathrm{e}$ de campo ${ }^{(7)}$. Essa técnica foi desenvolvida com base nos achados do trabalho clássico de Margaria et al.(8), no qual se demonstrou que logo após o exercício físico (2-3 min) o $\grave{V}_{2}$ se mantém elevado devido à ressíntese dos fosfatos de alta energia. Essa relação entre o $E P_{\text {RÁPIDO }} \mathrm{e}$ a ressíntese dos fosfatos de alta energia foi posteriormente confirmada em estudos realizados tanto em seres humanos ${ }^{(9)}$ como em animais $^{(10)}$. Como resultado, houve aumento da aplicação do $E P O C_{\text {RÁpiDo }}$ na determinação do MAA em diferentes situações ${ }^{(6,7)}$.

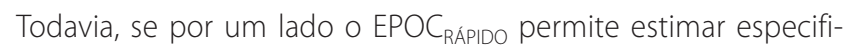
camente a participação do MAA, por outro lado os procedimentos envolvidos em sua determinação são relativamente complexos, pois envolvem a utilização de softwares específicos na modelagem matemática de curvas ${ }^{(6,7)}$. Essa modelagem matemática é empregada para estabelecer o tempo de duração do $\mathrm{EPOC}_{\text {RÁpiDO, }}$ haja vista que a resposta cinética do $\dot{\mathrm{V}}_{2}$ após o exercício físico pode possuir um comportamento mono ou biexponencial ${ }^{(11)}$. Infelizmente, a complexidade desses procedimentos pode desencorajar os treinadores e os cientistas do esporte a utilizarem o $E P O C_{\text {RÁpIDO }}$. Portanto, do ponto de vista prático, é desejável que métodos mais simples sejam desenvolvidos para aumentar a aplicabilidade do EPOC RÁPIDO.

A inspeção visual é uma das técnicas tradicionalmente empregadas na determinação de índices fisiológicos relacionados com o desempenho esportivo, tais como os limiares de lactato ou ventilatórios ${ }^{(12,13)}$. Portanto, é atraente a ideia de que seja possível a identificação visual do tempo de duração do EPOC RÁpIDo. Dessa forma, o objetivo do presente estudo foi analisar a validade, a reprodutibilidade e a objetividade do método de inspeção visual na identificação do $E P O C_{\text {RÁPIDO. }}$

\section{MÉTODOS}

\section{Amostra}

Dez indivíduos do sexo masculino, fisicamente ativos, com idade entre 20 e 35 anos e familiarizados com exercício exaustivo, participaram voluntariamente deste estudo após a leitura e assinatura do termo de consentimento livre e esclarecido. Todos os sujeitos estavam isentos de tratamentos farmacológicos, não eram fumantes e estavam livres de qualquer tipo de distúrbio neuromuscular ou cardiovascular. As características morfofuncionais dos participantes estão presentes na tabela 1. Os procedimentos adotados no presente estudo foram previamente aprovados pelo Comitê de Ética em Pesquisa local.
Tabela 1. Características morfofuncionais dos participantes $(n=10)$.

\begin{tabular}{|c|c|}
\hline Idade (anos) & $23,0 \pm 4,0$ \\
\hline Estatura $(\mathrm{cm})$ & $176,4 \pm 6,8$ \\
\hline Massa corporal (kg) & $72,4 \pm 8,2$ \\
\hline$\dot{\mathrm{V}} \mathrm{O}_{2} \max \left(L \cdot \mathrm{min}^{-1}\right)$ & $3,0 \pm 0,5$ \\
\hline$\dot{\mathrm{V}} \mathrm{O}_{2} \max \left(\mathrm{mL} \cdot \mathrm{kg}^{-1} \cdot \mathrm{min}^{-1}\right)$ & $41,5 \pm 5,7$ \\
\hline $\mathrm{WV}_{2} \max (\mathrm{W})$ & $250 \pm 40$ \\
\hline FCmax (bpm) & $179 \pm 9$ \\
\hline$\left[\mathrm{La}^{-}\right]$pico $\left(\mathrm{mmoL} \cdot \mathrm{F}^{-1}\right)$ & $10,28 \pm 1,42$ \\
\hline
\end{tabular}

Os valores são expressos em médias \pm desvios-padrão. $\dot{V}_{2}$ max = consumo máximo de oxigênio; $W_{\mathfrak{W}} \mathrm{O}_{2}$ max $=$ potência externa correspondente ao $\dot{\mathrm{V}}_{2} \max ; \mathrm{FCmax}=$ frequência cardíaca máxima; [La-]pico = concentraçöes sanguíneas de lactato de pico.

\section{Desenho experimental}

Todos os sujeitos foram submetidos a duas sessões experimentais que tiveram um intervalo mínimo de 72 horas e máximo de uma semana. Na primeira sessão foram realizadas as medidas antropométricas e um teste progressivo máximo em cicloergômetro para a mensuração do $\dot{\mathrm{V}} \mathrm{O}_{2}$ max e da potência externa atingida no $\dot{\mathrm{V}}_{2} \max \left(\mathrm{W} \dot{\mathrm{V}} \mathrm{O}_{2}\right.$ max).Na segunda sessão foi realizado um teste de carga constante com a intensidade de $110 \%$ da $\mathbf{W V}_{2}$ max para determinar O EPOC RÁPIDO. Os testes foram realizados com a temperatura ambiente semelhante $\left(20-24^{\circ} \mathrm{C}\right)$ e duas horas após a última refeição. Os sujeitos foram solicitados a não praticar exercícios físicos extenuantes e a não ingerir álcool ou substâncias que possuíssem cafeínanas 48 horas que antecederam as coletas dos dados.

\section{Teste progressivo máximo}

O teste progressivo máximo foi realizado em cicloergômetro eletromagnético de membros inferiores (Godart-Holland, Lannoy). A altura do selim foi individualmente ajustada, tendo como referência a extensão quase total dos joelhos dos sujeitos em um ciclo completo do pedal. Após um aquecimento de três minutos utilizando apenas a resistência inercial do equipamento, a intensidade de esforço foi aumentada em $30 \mathrm{~W} \cdot \mathrm{min}^{-1}$ até a exaustão voluntária. Os indivíduos foram orientados a manter a rotação do pedal em 60 rpm durante todo o teste. O teste foi interrompido quando o individuo não conseguia manter a cadência do pedal acima de $50 \mathrm{rpm}$. Durante todo o teste as trocas gasosas e a frequência cardíaca $(F C)$ foram mensuradas respiração a respiração. Amostras de sangue arterializado foram coletas do lóbulo da orelha imediatamente após o teste, no segundo e no terceiro minutos da recuperação para mensurar as concentrações sanguíneas de lactato de pico([La]pico). A frequência cardíaca máxima (FCmax) foi estabelecida pelo maior valor medido ao final do teste. A média do consumo de oxigênio nos 30 segundos finais do último estágio completado foi utilizada para representar o $\dot{\mathrm{V}}_{2}$ max. $\mathrm{A} \mathbf{W}_{\dot{V}} \mathrm{O}_{2}$ max foi estabelecida como a potência externa máxima em que o $\dot{\mathrm{V}}_{2}$ max foi alcançado.

\section{Teste com carga constante}

O cicloergômetro, o ajuste da altura do selim, a cadência do pedal, o aquecimento, o critério de interrupção e a mensuração do $\dot{V}_{2}$ no exercício com carga constante foram idênticos aos empregados no teste progressivo até a exaustão. Antes do início do teste, os sujeitos permaneceram cinco minutos sentados no selim do cicloergômetro para a mensuração da linha de base do $\dot{V}_{2}$. Logo após, os participantes se exercitaram até a exaustão voluntáriana intensidade de $110 \%$ da Wvن ${ }_{2} \max$, seguindo-se dez minutos de recuperação passiva com o individuo sentado no selim do cicloergômetro. Os participantes foram instruídos a não realizar movimento com braços e pernas durante a fase 
de recuperação. A média do $\dot{V}_{2}$ nos 30 segundos finais do exercício foi utilizada para representar o valor de pico do $\dot{V}_{2}$. O pico da FC foi medido ao final do teste. Além disso, amostras contendo $25 \mu \mathrm{L}$ de sangue arterializado foram coletadas do lóbulo da orelha imediatamente após o término do exercício, no terceiro, no quinto e no sétimo minutos de recuperação para a mensuração das [La']pico.

\section{Coleta dos dados fisiológicos}

$\mathrm{O} \dot{\mathrm{V}} \mathrm{O}_{2}$ foi mensurado continuamente respiração a respiração por meio de um analisador de gases portátil ( $K 4 b^{2}$ Cosmed, Roma, Itália), ao passo que a FC foi medida por um cardiofrequencímetro (Polar, Kempele, Finlândia) acoplado a esse equipamento. Uma máscara de silicone com baixa resistência que mantém o nariz e a boca cobertos (Hans Rudolph, Kansas City, EUA) foi empregada na coleta do ar utilizado nas análises da ventilação, das frações do oxigênio e do dióxido de carbono. Antes de cada sessão de coleta de dados, o analisador de gases foi calibrado utilizando o ar ambiente e um cilindro de gás de composição conhecida (20,9\% de $\mathrm{O}_{2}$ e $5 \%$ de $\mathrm{CO}_{2}$ ). A calibração do fluxo de ar foi realizada com uma seringa com volume de três litros (Quinton Instruments, Seattle, WH). A calibragem do tempo de atraso para a análise da amostra de ar expirada foi realizada de acordo com as especificações do fabricante ( $K 4 b^{2}$ instruction manual). Esse tempo de atraso é de aproximadamente $500 \mathrm{~ms}$ e automaticamente considerado nos cálculos das trocas gasosas. As concentrações sanguíneas de lactato foram determinadas por meio de um analisador automático de lactato (Yellow Springs 1500 Sport, Ohio, EUA), o qual foi calibrado a cada dez análises, tendo como referência a concentração de lactato de $5 \mathrm{mmol} \cdot \mathrm{L}^{-1}$.

\section{Métodos de determinação do $\mathrm{EPOC}_{\mathrm{RÁPIDO}}$ Método matemático}

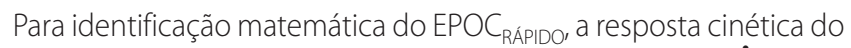
$\dot{\mathrm{V}}_{2}$ após o exercício executado na intensidade de $110 \%$ da $\mathbf{W V}_{2}$ max foi individualmente ajustada por um modelo biexponencial (equação 1), conforme descrito previamente ${ }^{(11)}$ (Origin, Microcal, EUA). Logo após, a equação 2 foi aplicada no intuito de se determinar o EPOC RÁPIDO $^{(6,7)}$.

$$
\begin{array}{ll}
\dot{\mathrm{V}} \mathrm{O}_{2(t)}=y 0+\left(\mathrm{A}_{1} \mathrm{e}^{-(t-\delta) / \tau_{1}}\right)+\left(\mathrm{A}_{2} \mathrm{e}^{-(t-\delta) / \tau} \tau_{2}\right) & \text { Equação1 } \\
\mathrm{EPOC}_{\text {RAPPDO }}=\mathrm{A}_{1} \cdot \tau_{1} & \text { Equação2 }
\end{array}
$$

Onde ${ }_{1} e_{2}$ denotam, respectivamente, o componente rápido e lento do excesso do consumo de oxigênio após o exercício; yo = linha de base do consumo de oxigênio; $A, \tau$ e $\delta$ são referentes à amplitude em $\mathrm{mL} \cdot \mathrm{min}^{-1}$, à constante de tempo (s) e ao tempo de atraso (s), respectivamente.

\section{Método visual}

As respostas do $\dot{V}_{2}$ após o exercício de carga constante foram graficamente apresentadas em um software (Microsoft Office Excel2003) a três avaliadores que possuíam experiência na determinação de índices bioenergéticos relacionados com o desempenho esportivo. Nesses gráficos $\circ \dot{\mathrm{V}} \mathrm{O}_{2}$ foi expresso em $\mathrm{mL} \cdot \mathrm{min}^{-1}$ e plotado sobre o eixo das ordenadas, ao passo que o tempo foi expresso em segundos e plotado sobre eixo das abscissas. Os avaliadores receberam uma breve informação acerca da relação existente entre o $E P O C_{\text {RápiDo }}$ e a ressíntese dos fosfatos de alta energia, garantindo que todos eles possuíssem informações básicas acerca do EPOC RÁpido. Em duas ocasiões separadas, os avaliadores detectaram visualmente o tempo de duração do $\mathrm{EPOC}_{\text {RÁpIDO. }}$ Isso foi realizado com o cursor do computador sobre o $\dot{\mathrm{V}}_{2}$, o que permitiu identificar o tempo apresentado no eixo das ordenadas. Dessa forma, o consumo de oxigênio acumulado até o ponto no tempo de- signado pelos avaliadores foi calculado pelo somatório dos valores de $\dot{\mathrm{V}} \mathrm{O}_{2}$. Posteriormente, a linha de base do $\dot{\mathrm{V}}_{2}$ foi subtraída do consumo de oxigênio acumulado para a determinação do PPOC $_{\text {RÁpiDo. No intuito }}$ de analisar a reprodutibilidade do método visual, cada avaliador realizou duas vezes esse procedimento de identificação visual do $E P O C_{\text {RÁpIDO }}$ com um intervalo mínimo de 72h e máximo de uma semana.

\section{Análises estatísticas}

Todas as análises estatísticas foram feitas utilizando o programa computadorizado SPSS (versão 13.0, Chicago, EUA). Os dados foram reportados como médias e desvios-padrão (SD). Para verificar a validade do método visual, os valores gerados pelos três avaliadores foram comparados com o método matemático por ANOVAone way. A plotagem de Bland e Altman também foi empregada para a análise da diferença entre os dois métodos. O coeficiente de correlação de Pearson foi utilizado para verificar o grau de associação entre os dois métodos. A ANOVAone way foi utilizada para analisar a objetividade do método visual comparando os resultados gerados pelos avaliadores. Areprodutibilidade foi analisada pelo coeficiente de correlação intraclasse (ICC). O nível de significância de 5\% $(p<0,05)$ foi adotado em todas as análises.

\section{RESULTADOS}

A intensidade de $110 \%$ da $\mathbf{W V}_{2}$ max correspondeu a uma potência externa de $276 \pm 43 \mathrm{~W}$. O tempo de duração do exercício realizado nessa intensidade foide $161 \pm 41$ segundos. Os valores de pico do $\dot{\mathrm{V}} \mathrm{O}_{21}$ da $\mathrm{FC}_{\text {MAX }}$ e das [La] $\mathrm{Lico}$ foramde $2.706,0 \pm 499 \mathrm{~mL} \cdot \mathrm{min}^{-1}, 174 \pm 13 \mathrm{bpm}$ e10,9 $\pm 1,2 \mathrm{mmoL} \cdot L^{-1}$, respectivamente.

A linha de base do $\dot{\mathrm{V}} \mathrm{O}_{2}$ foi de $278,4 \pm 57,1 \mathrm{~mL} \cdot \mathrm{min}^{-1}$, ao passo que os valores da constante de tempo e da amplitude utilizados no cálculo do $E_{P O C_{\text {RAPIDO }}}$ foram de $44 \pm 4$ segundos e 1.352,7 $\pm 325,5=\mathrm{mL} \cdot \mathrm{min}^{-1}$, respectivamente. A figura 1 demonstra um exemplo do comportamento típico da resposta do $\dot{V} \mathrm{O}_{2}$ após o exercício em $110 \%$ da $\mathbf{W V}_{2}$ max e o tempo de duração do $E P O C_{\text {RÁplDo }}$ identificado visualmente pelos três avaliadores.

Nenhuma diferença significativa foi detectada entre os valores do EPOC RÁPIDO determinados pelo método matemático e visual (Figura 2). Do mesmo modo, os outros parâmetros derivados do método visual também não foram diferentes entre os avaliadores (Tabela 2). A plo-

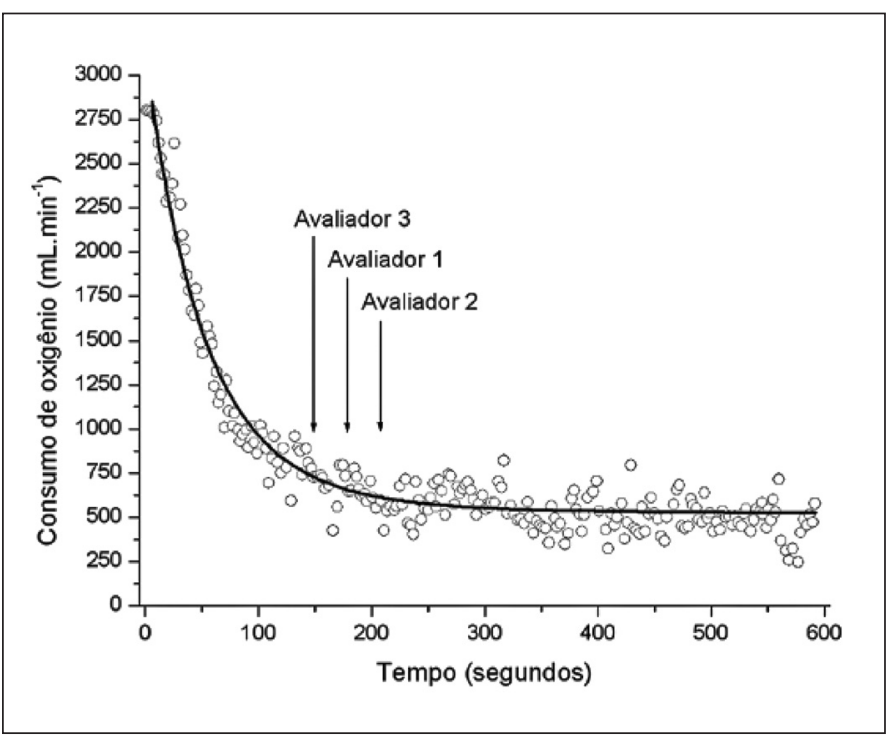

Figura 1. Comportamento típico da resposta do consumo de oxigênio após o exercício

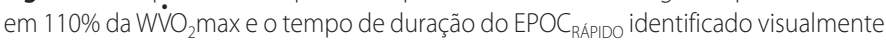
pelos três avaliadores. A linha central representa os valores médios gerados pelo ajuste matemático. As setas indicam a identificação feita por cada avaliador. 
tagem de Bland e Altman revelou que todos os valores gerados pelo método visual encontravam-se no intervalo de confiança de 95\% ( \pm 1,96 desvio-padrão), conforme ilustrado na figura 3. Adicionalmente, os resultados do EPOC $_{\text {RÁPIDO }}$ obtidos pelos diferentes métodos e avaliadores também foram significantemente correlacionados (Tabela 3).

Na reavaliação utilizada para analisar a reprodutibilidade do método

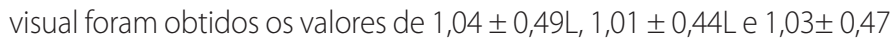
do EPOC $_{\text {RÁPIDO }}$ para os avaliadores 1, 2 e 3, respectivamente, os quais não foram significativamente diferentes dos valores encontrados na primeira avaliação (1,04 $\pm 0,45 \mathrm{~L} ; 1,02 \pm 0,45 \mathrm{~L} ; 1,02 \pm 0,45 \mathrm{~L}$, respectivamente). 0 ICC obtido nas duas identificações visuais do EPOC $C_{\text {RÁPIDO }}$ dos avaliadores 1,2 e 3 foide 0,98, 0,99 e 0,97 ( $p<0,05$ ), respectivamente.

\section{DISCUSSÃO}

A possibilidade de estimar a contribuição do MAA em situações laboratoriais(6) e de campo $^{(7)}$ é uma das principais vantagens do EPOC $_{\text {RÁPIDO }}$ Contudo, a relativa complexidade envolvida nos procedimentos matemáticos empregados no cálculo do EPOC RápIDo pode

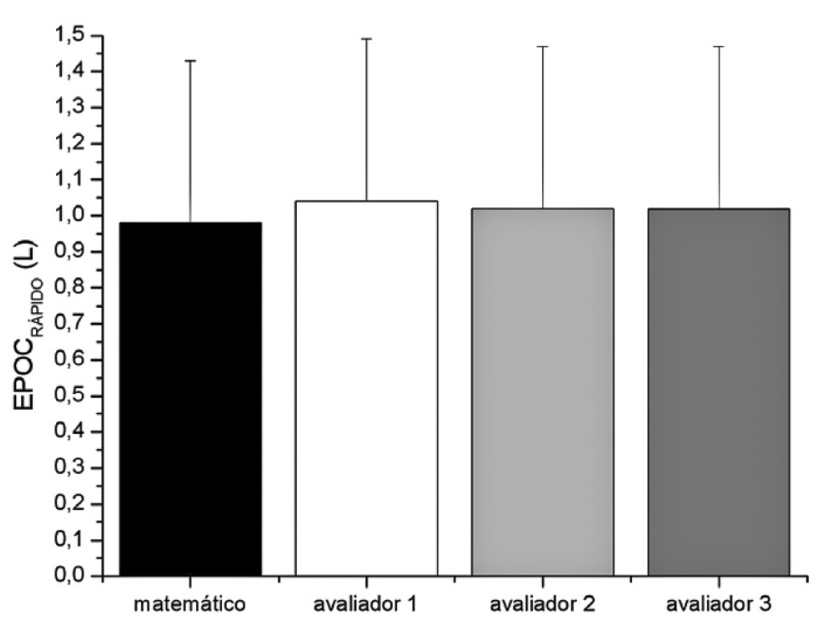

Figura 2. Fase rápida do excesso do consumo de oxigênio após o exercício (EPOC RÁPIDO determinado pelos métodos matemático e visual $(n=10)$.

Tabela 2. Parâmetros da fase rápida do excesso do consumo de oxigênio após o exercício, os quais foram estabelecidos a partir do método visual pelos três avaliadores $(n=10)$.

\begin{tabular}{l|c|c|c}
\hline & Avaliador 1 & Avaliador 2 & Avaliador 3 \\
\hline Tempo de duração $(s)$ & $139 \pm 24$ & $146 \pm 38$ & $131 \pm 12$ \\
\hline$\dot{\mathrm{V}} \mathrm{O}_{2}$ acumulado $(\mathrm{L})$ & $1,69 \pm 0,57$ & $1,71 \pm 0,54$ & $1,63 \pm 0,48$ \\
\hline Linha de base do $\dot{\mathrm{V}} \mathrm{O}_{2}(\mathrm{~L})$ & $0,65 \pm 0,20$ & $0,69 \pm 0,24$ & $0,61 \pm 0,48$ \\
\hline$\dot{\mathrm{V}}_{2}=$ consumo de oxigênio expresso em litros de $\mathrm{O}_{2}$.
\end{tabular}

Tabela 3. Correlações da fase rápida do excesso do consumo de oxigênio após o exercício determinada pelos métodos matemático e visual, sendo o último método estabelecido por três avaliadores $(n=10)$.

\begin{tabular}{l|c|c|c|c}
\hline & Matemático & Avaliador 1 & Avaliador 2 & Avaliador 3 \\
\hline Matemático & - & $0,83^{* *}$ & $0,75^{*}$ & $0,71^{*}$ \\
\hline Avaliador 1 & - & - & $0,99^{* *}$ & $0,98^{* *}$ \\
\hline Avaliador 2 & - & - & - & $0,99^{* *}$ \\
\hline Avaliador 3 & - & - & - & - \\
\hline
\end{tabular}
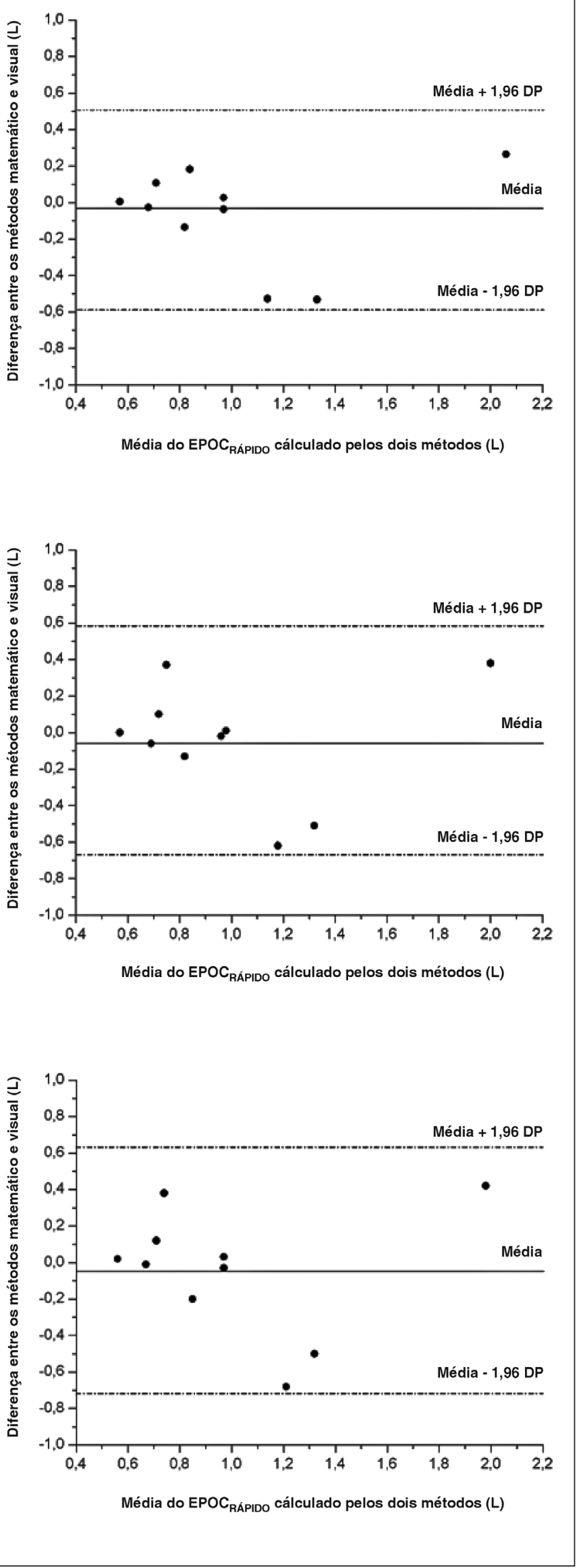

Figura 3. Plotagem de Bland e Altman para os valores da fase rápida do excesso do consumo de oxigênio após o exercício (EPOC $C_{\text {RÁPDO }}$ ), a qual foi estabelecida pelo método matemático e visual $(n=10)$. O painel superior refere-se ao avaliador 1 , o painel intermediário ao avaliador 2 e o painel inferior ao avaliador 3. 
desencorajar a sua utilização. Nesse sentido, o objetivo do presente estudo foi analisar a validade, a reprodutibilidade e a objetividade do método de inspeção visual aplicado à identificação do EPOC RÁPIDO. Os principais achados foram: a) os valores do $E_{P O C_{R A ́ P I D O}}$ gerados pelos dois métodos não eram estatisticamente diferentes; b) de modo similar, os valores do EPOC $_{\text {RÁPIDO }}$ estabelecidos visualmente também não eram diferentes entre os três avaliadores; c) os resultados do ICC sugerem que o método visual é altamente reprodutível.

Durante os exercícios de alta intensidade, a manutenção dos processos de contração muscular ocorre predominantemente por vias que não necessitam de $\mathrm{O}_{2}$, denominadas de metabolismo anaeróbio. O sistema anaeróbio, por sua vez, é subdividido em componente alático e lático, os quais se referem, respectivamente, à hidrólise dos estoques dos fosfatos de alta energia (ATP e CP) e à degradação parcial da glicose, resultando na formação de ácido láctico(1). Do ponto de vista temporal, acredita-se que a contribuição dos metabolismos anaeróbios é de $\sim 80 \%, \sim 45 \%$ e $\sim 30 \%$ da energia total nos exercícios cíclicos e de alta intensidade que têm a duração de 30 segundos, entre 60 e 90 segundos e entre 120 e 180 segundos, respectivamente ${ }^{(14)}$. Assim, ao se analisar a relação entre o tempo e a intensidade dos exercícios, supõe-se que esse metabolismo desempenha papel fundamental em algumas tarefas contínuas ${ }^{(15)}$ e intermitentes ${ }^{(14)}$. Consequentemente, diversos testes foram propostos para avaliarem os metabolismos anaeróbios a partir de índices mecânicos (por exemplo, teste de Wingate) e fisiológicos (por exemplo, déficit de oxigênio) ${ }^{(5)}$.

Tem-se sugerido que a validade de novos testes possa ser analisada mediante a comparação entre grupos de indivíduos com diferentes estados de treinamento (validação por constructo), com um valor de referência (validade por critério) ou a partir da opinião de especialistas (validação lógica) ${ }^{(16)}$. No presente estudo, empregou-se o método de validação por critério, assumindo que o método matemático é o padrão-ouro para a determinação do EPOC RÁPIDO. Investigações prévias utilizaram esse método para estabelecer o $E P O C_{\text {RÁPIDO }}$ devido à necessidade de se determinar o tempo de duração da fase rápida da resposta cinética do $\dot{V}_{2}$ após o exercício ${ }^{(6,7)}$. Os nossos resultados demonstram que a diferença entre o método matemático e visual foi inferior a 6\%. Esses achados têm importante aplicação prática, haja vista que o método de inspeção visual reduz substancialmente a complexidade dos procedimentos empregados no estabelecimento do EPOC $_{\text {RÁPIDO. Dessa forma, }}$ o método de inspeção visual poderá auxiliar futuros trabalhos que eventualmente tenham como objetivo quantificar a contribuição do MAA durante o exercício de alta intensidade de modo mais simples.

A compreensão dos procedimentos necessários para a aplicação de novos testes é uma etapa importante no processo de validação. Isso se faz necessário para que outros pesquisadores possam replicar tais procedimentos e assim garantir que os resultados obtidos não sofram influência de outras variáveis externas. Na presente investigação, a objetividade do método visual de determinação do EPOC RÁPIDO foi analisada por três avaliadores que possuíam experiência na determinação de índices fisiológicos. Nossos resultados revelam que não houve diferenças entre os valores do EPOC RÁPIDO obtidos pelos três avaliadores, indicando que os procedimentos envolvidos no método visual são de fácil compreensão, desde que os avaliadores possuam alguma experiência na determinação de índices fisiológicos.

A reprodutibilidade tem sido indicada como um parâmetro importante para o estabelecimento de variações biológicas ou técnicas de análise e deve ser estabelecida antes que um novo procedimento seja proposto ${ }^{(16)}$. Ela pode ser conhecida de diferentes formas, porém, quando o ICC é utilizado, valores entre 0,70 e 0,80 devem ser vistos com cautela, ao passo que valores maiores que 0,90 podem ser considerados altamente reprodutíveis ${ }^{(16)}$. Como foram encontrados valores de ICC entre 0,97 e 0,99, é plausível sugerir que o método proposto no presente estudo é consistente em termos de reprodutibilidade. Isso reforça a sua aplicabilidade em futuros trabalhos que desejem quantificar a contribuição do MAA durante o exercício intenso.

Em resumo, os achados do presente estudo sugerem que a inspeção visual é um método válido para a determinação do EPOC $C_{\text {ŔPIDO }}$. Além disso, esse método é altamente reprodutível e de fácil compreensão para pessoas que possuem alguma experiência na identificação de índices fisiológicos relacionados com odesempenho esportivo.

\section{AGRADECIMENTOS}

Os autores agradecem a participação de todos os sujeitos. Rômulo Cássio de Moraes Bertuzzi é bolsista da Fundação de Amparo à Pesquisa do Estado de São Paulo (processo 2008/50934-1).

Todos os autores declararam não haver qualquer potencial conflito de interesses referente a este artigo.

\section{REFERÊNCIAS}

1. Gastin PB. Energy system interaction and relative contribution during maximal exercise.Sports Med 2001;3:725-41.

2. di Prampero PE, Ferretti G. The energetics of anaerobic muscle metabolism: a reappraisal of older and recent concepts.Respir Physiol 1999;118:103-15.

3. Paavolainen L, Nummela A, Rusko H. Muscle power factors and $\mathrm{VO}_{2}$ max as determinants of horizontal and uphill running performance.Scand J Med Sci Sports2000;10:286-91.

4. Bulbulian R, Wilcox AR, Darabos BL. Anaerobic contribution to distance running performance of trained cross-country athletes.Med Sci Sports Exerc 1986;18:107-13.

5. Green S, Dawson B. Measurement of anaerobic capacities in humans. Definitions, limitations and unsolved problems.Sports Med 1993;15:312-27.

6. Beneke R, Pollmann C, Bleif I, Leithäuser RM, Hütler M. How anaerobic is the Wingate Anaerobic Test for humans? Eur J Appl Physiol 2002;87:388-92.

7. Beneke R, Beyer T, Jachner C, Erasmus J, Hütler M. Energetics of karate kumite.Eur J Appl Physiol 2004;92:518-23.

8. Margaria R, Edwards HT, Dill DB. The possible mechanism of contracting and paying the oxygen debtand the role of lactic acid in muscular contraction. Am J Physiol 1933;106:689-714.
9. Haseler $L$, Hogan MC, Richardson RS. Skeletal muscle phosphocreatine recovery in exercise-trained humans is dependent on $\mathrm{O}_{2}$ availability.J Appl Physiol 1999;86:2013-8.

10. Piiper J, Spiller P. Repayment of $\mathrm{O}_{2}$ debt and resynthesis of high-energy phosphates in gastrocnemius muscle of the dog.J Appl Physiol 1970;28:657-62.

11. Ozyener F, Rossiter HB, Ward SA, Whipp BJ. Influence of exercise intensity on the on- and off-transient kinetics of pulmonary oxygen uptake in humans.J Physiol 2001;533:891-902.

12. Caiozzo VJ, Davis JA, Ellis JF, Azus JL, Vandagriff R, Prietto CA, McMaster WC. A comparison of gas exchange indices used to detect the anaerobic threshold.J Appl Physiol 1982;53:1184-9.

13. Bischoff MM, Duffin J. An aid to the determination of the ventilatory threshold. Eur J Appl Physiol Occup Physiol 1995;71:65-70.

14. Spriet, LL. Anaerobic metabolism during high-intensity exercise. In: HARGREAVES, M, editors. Exercise metabolism. Champaign: Human Kinetics;1995. p.1-39

15. Karvonen J, Peltola E, Näveri H, Härkönen M. Lactate and phosphagen levels in muscle immediately after a maximum $300 \mathrm{~m}$ run at sea level.Res Q Exerc Sport 1990;61:108-10.

16. Currell $K$, Jeukendrup AE. Validity, reliability and sensitivity of measures of sporting performance. Sports Med 2008;38:297-316. 